\title{
The effect of dust scattering on the timing properties of black holes
}

\author{
Emrah Kalemci* \\ Sabancı University, Orhanl-- Tuzla, Istanbul, TURKEY \\ E-mail: ekalemciesabanciuniv.edu \\ John A. Tomsick \\ SSL, UC Berkeley \& CASS, UC San Diego, CA, USA. \\ E-mail: jtomsickessl.berkeley.edu
}

Richard E. Rothschild

CASS, UC San Diego, CA, USA.

E-mail: rrothschildeucsd.edu

David M. Smith

SCIPP, UC Santa Cruz, CA, USA.

E-mail: dsmithescipp.ucsc.edu

\begin{abstract}
It has been known that sources with high absorption column density also have high dust column density along the line of sight. The differential delays caused by small angle scattering of $\mathrm{X}$ rays by dust may have important effects on the power spectra of Galactic black holes at low energies, and impact studies that use the relation between the rms amplitude of variability and energy to determine the origin of QPOs from these sources. We observed the high absorption column density $\left(N_{H} \sim 10^{23} \mathrm{~cm}^{-2}\right)$ GBH 1E1740.7-2942 for $20 \mathrm{ks}$ simultaneously with XMM-Newton and RXTE. By comparing the power spectra from the events in the core of the point spread function (PSF) of XMM-Newton EPIC-PN (using imaging and excluding the scattering halo) and the RXTE data, we quantified the effects of small angle scattering on the timing properties of this source. The rms amplitude of variability in $\sim 2-6 \mathrm{keV}$ band obtained from the XMM-Newton data is higher than that of the RXTE as expected from the a scattering halo contribution in the RXTE.
\end{abstract}

VI Microquasar Workshop: Microquasars and Beyond

September 18-22 2006

Società del Casino, Como, Italy

* Speaker. 


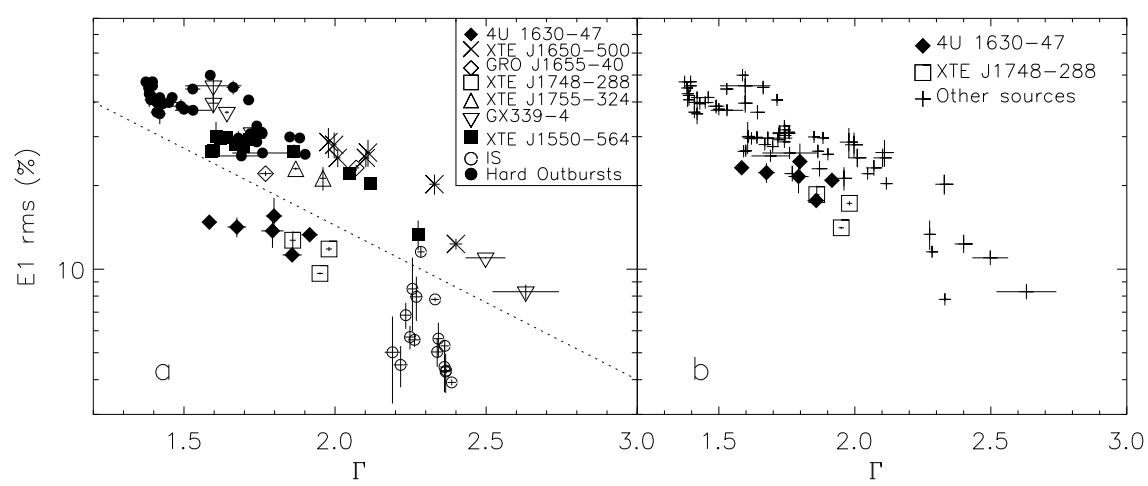

Figure 1: (a) The correlation between the spectral index and the rms amplitudes in 2-6 keV band (E1). The hard outbursts and the IS observations are shown with one symbol. The dashed line is to guide the eye to the correlation above it that exists for all observations except the IS, 4U 1630-47 and XTE J1748-288 observations. From [Kalemci 2002]. (b) The correlation between the photon index and the rms amplitudes in 2-6 keV band, with dust scattering correction applied for 4U 1630-47 and XTE J1748-288. All other sources are shown with crosses.

\section{Introduction}

It is known that many global correlations exist between spectral and temporal properties of Galactic black holes (GBHs) [Kalemci 2002]. One example is between the rms amplitude of variability and the spectral index (Fig. 1). The intermediate state (IS) and the observations of $4 \mathrm{U}$ 1630-47 and XTE J1748-288 do not obey the correlation. The low amplitude of IS observations can be explained by a non-varying accretion disk [Kalemci et al. 2004]. Two low rms amplitude sources, 4U 1630-47 and XTE J1748-288 have very high hydrogen absorption column densities compared to the other sources, $N_{H}>7 \times 10^{22} \mathrm{~cm}^{-2}$. There is other evidence that links $N_{H}$ to the timing properties of black holes. All high $N_{H}$ sources show an increasing rms amplitude of variability with energy. Some of the sources that show increasing rms amplitude as a function of energy are 4U 1630-47, XTE J1748-288, GRS J1737-31, 1E 1740.7-2942 which all have $N_{H}$ between (6-9.5) $\times 10^{22} \mathrm{~cm}^{-2}$ [Kalemci 2002, Lin et al. 2000].

The reduced rms amplitude of variability at low energies may be due to the presence of a scattering halo [Kalemci 2002]. There is a strong correlation between the dust $\left(\tau_{s c a}\right)$ and the neutral hydrogen column densities; $\tau_{\text {sca }}=0.49 \times\left(N_{H} /\left(10^{22} \mathrm{~cm}^{-2}\right)\right) \times(E / \mathrm{keV})^{-2}$ [Predehl \& Schmitt 1995]. Therefore, all of the sources with high $N_{H}$ are behind copious amount of cosmic dust which results in small angle scattering of the $\mathrm{X}$ rays. The scattering cross section scales as $\sim 1 / E^{2}$ where $E$ is the energy of the X-ray photon. $\mathrm{X}$ rays scattered back into the line of sight travel along longer paths than the unscattered $X$ rays, and the corresponding lags may cause loss of coherence and a decrease in the rms amplitude of variability, if the entire halo is in the field of view of the instrument, like RXTE.

If spherical dust grains of radius $a$ are evenly distributed along the line of sight, the delay is on the order of days [Molnar \& Mauche 1986]. Since the dominant variability timescales are between ms to tens of seconds in GBHs, the scattered X rays would leave the shape of the power spectrum intact, but would reduce its rms amplitude. For example, although the total rms amplitude increases with energy for 4U 1630-47 and XTE J1748-288, the shape of the power spectra remain constant. 
There are several consequences of this effect. For black hole transients, this could mean that there is a strong, global correlation between the photon index and the "intrinsic" rms amplitude of variability, valid for all sources. Using $N_{H}$ values of 9 and $7.5 \times 10^{22} \mathrm{~cm}^{-2}$, we estimated that $36 \%$ and $32 \%$ of the total flux is in the scattering halo in $2-6 \mathrm{keV}$ band for $4 \mathrm{U} 1630-47$ and XTE J1748-288 respectively. Factoring those in for the rms amplitude, and placing the points back into the photon index - rms amplitude of variability relation restores the global correlation valid for all sources (see Fig. 1.b).

The studies that use the relation between the rms amplitude of variability and the energy to determine the physical origin of the QPOs and where they are created [Tomsick \& Kaaret. 2004, Rodriguez et al. 2004] must take this effect into account for high $N_{H}$ sources.

The study of the effect of dust scattering on timing could also yield important results in terms of determining the properties of the dust in the interstellar medium (ISM), such as its density distribution along the line of sight [Molnar \& Mauche 1986, Predehl \& Schmitt 1995]. If the dust column density is concentrated in one cloud close to the source, the observed lags may be much smaller. This would alter the shape of the power spectrum.

To test the idea that the high $N_{H}$ sources timing characteristics may be affected by dust scattering we observed black hole 1E1740.7-2942 simultaneously with XMM-Newton and RXTE. For reasonable grain size and dust densities, more than $99 \%$ of the halo emission is within $\sim 25^{\prime}$ of the source [Predehl \& Klose 1996]. This means the entire scattering halo is within the 1 degree field of view of RXTE. On the other hand, the overwhelming majority (>99\%) of the photons in the core of the PSF (15“ diameter) of the XMM-Newton EPIC-PN would be unscattered X rays. In this work, we compared the PSDs from two instruments in 2-6 keV band, and quantified the effect of the scattering halo on timing properties of 1E1740.7-2942.

\section{Observations and Analysis}

We have chosen 1E1740.7-2942 for three reasons: 1 . it is known to have very high $N_{H}(1.2 \times$ $10^{23} \mathrm{~cm}^{-2}$ determined by XMM-Newton), 2. it is a persistent source known to be in the low hard state most of the time, 3. extended emission has been detected from the source by Chandra X-ray Observatory [Cui et al.2001]. Moreover, the typical count rate from the source is high enough to do timing and at the same time low enough to avoid pile-up effects in XMM-Newton EPIC-PN. Even though the source has these desirable properties, it also has 2 disadvantages, 1. a strong source nearby requires offset pointing with $R X T E, 2$. there is very strong diffuse ridge emission.

1E1740.7-2942 was observed for $\sim 20$ ks simultaneously with XMM-Newton and RXTE on MJD 53645. With XMM-Newton, we used small window mode for EPIC-PN and timing mode for EPIC-MOS. EPIC-PN with standard cuts and observing region limited to 15 " yields a rate of $\sim 9$ cts/s. The image of the source and the PSF is shown in Fig. 2. We used the events inside the black circle shown in Fig. 2 to obtain a light curve in the 2.11-5.86 keV band using XMM-SAS, and obtained a power spectrum with rms normalization. The background is negligible.

The effect of dead-time is simulated as follows. We generated light curves with different mean count rates, and then cut the electronic read-out times effectively creating zero count windows (1.7 $\mathrm{ms}$ for each $5.7 \mathrm{~ms}$ frame) in the light curve. Then we obtained Leahy normalized power spectra from these light curves. The resulting power spectra was flat and averaged at 1.996 regardless 


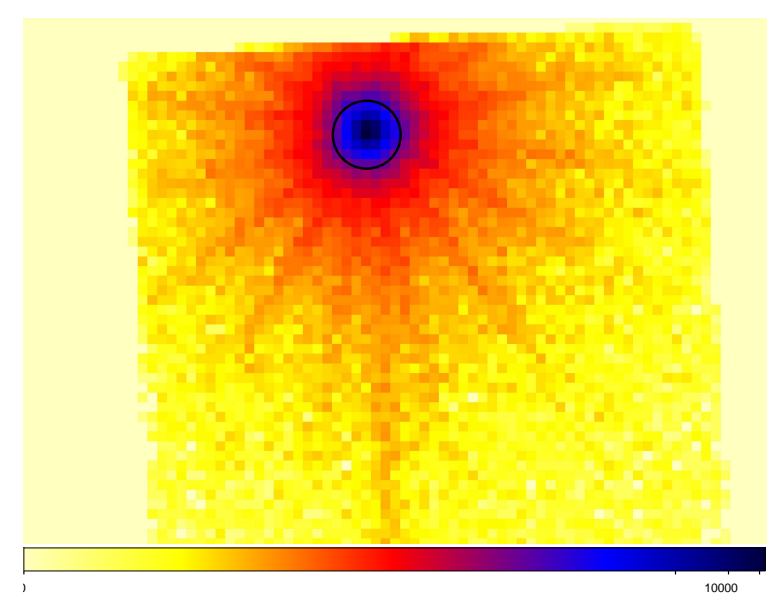

Figure 2: $X M M-N e w t o n$ EPIC-PN image in 2-6 keV band, and the area we used to obtain the light curve.

of the count rate. We subtracted 1.996 from the PSD instead of the nominal value of 2 for rms normalization.

With RXTE, we obtained $26 \mathrm{cts} / \mathrm{s}$ (after the subtraction of the ridge) in 2-6 keV band using PCA. We used FTOOLS to obtain the light curve in 2.11-5.86 keV band and calculated the power spectrum. For $R X T E$, we multiplied the rms amplitude of variability with $\frac{T^{2}}{(T-(R+B))^{2}}$, where $T$ is the overall count rate, $B$ is the instrumental background rate, and $R$ is the count rate due to the Galactic ridge, to obtain the variability inherent to the source [Berger \& van der Klis 1994]. The count rate due to the ridge emission is determined using the earlier offset background pointings of the source. The effective area was taken into account while determining the count rates. For more information on the offset pointing for the source and the background, see Main et al., 1999.

\section{Results and discussion}

The simultaneous power spectra in the 2.11-5.86 keV band is shown in Fig. 3 along with zero centered Lorentzian fits. The rms amplitude of variability from the XMM-Newton PSD is $15.73 \pm$ $1.36 \%$ with a peak frequency of $1.1 \pm 0.3 \mathrm{~Hz}$. On the other hand, the rms amplitude of variability from the RXTE is $12.27 \pm 1.21 \%$, peaking at $2.4 \pm 0.7 \mathrm{~Hz}$. The XMM-Newton EPIC-PN effective area is flat between 2.11 and $5.86 \mathrm{keV}$ bands. On the other hand, PCA effective area sharply increases. Therefore it is possible that the discrepancy is even higher between the rms amplitudes if we take the effective areas into account, as less low energy photons are collected with the RXTE.

For a uniform distribution, just a change in the rms amplitude is expected. If the dust is concentrated close to the source, the scattering may result in loss of high frequency variability as the time delays are shorter. In this case we expect not only less rms variability from the RXTE PSD, but also a smaller peak frequency. On the other hand, the RXTE peak frequency is higher than that of XMM-Newton. The quality of the PSDs prevented a robust comparison of their shapes as the high frequency part of the PSDs is very noisy. The rms and the peak frequencies are within $2 \sigma$.

We plan to characterize the halo emission by spectral means as well by comparing the XMMNewton and the RXTE spectra. We plan to use MOS Timing data for an additional way of cal- 


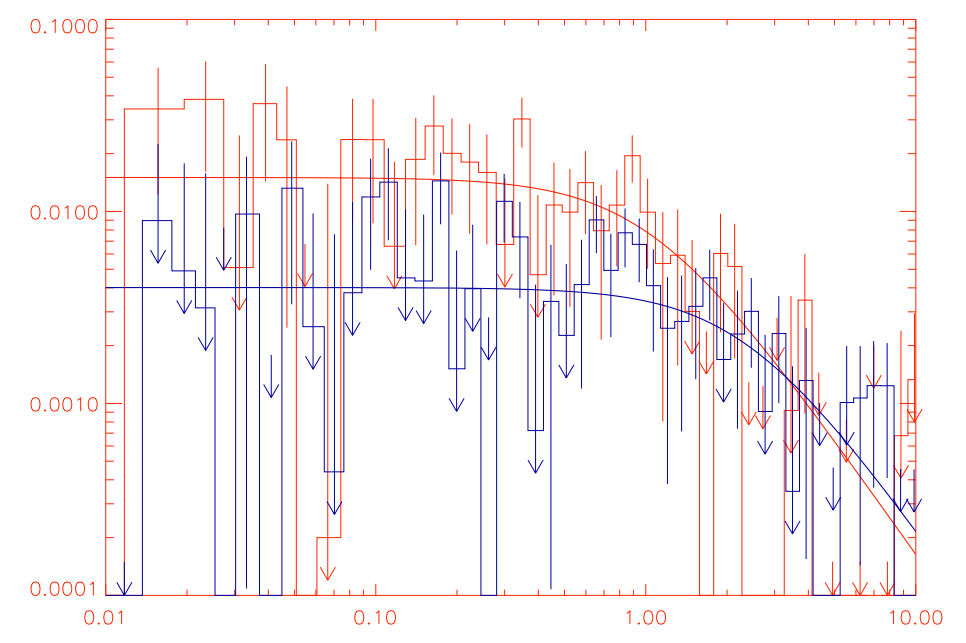

Figure 3: $X M M$-Newton Core of the PSF (red) and RXTE (blue) PSD of 1E1740.7-2942 after all corrections in $2.11-5.86 \mathrm{keV}$ bands. The zero centered Lorentzian fits are over-plotted. Long error bars are truncated with arrows for clarity.

culating the rms amplitude of variability from the XMM-Newton data, and also plan to extend the comparison to higher energy bands taking the effective area considerations fully into account.

\section{Acknowledgments}

E.K. is supported by the European Commission through a FP6 Marie-Curie International Reintegration Grant (INDAM). E.K. acknowledges partial support of TÜBİTAK. This project is supported by NASA Grant NNG06GB90G. EK thanks Dr. Uttley for fruitful discussions on the effective area of the instruments and Tommy Thompson for the discussion on dust scattering in general.

\section{References}

[Berger \& van der Klis 1994] Berger, M., \& van der Klis, M., 1994, A\&A, 292, 175

[Cui et al.2001] Cui, W. et al., 2001, ApJ, 548, 394

[Kalemci 2002] Kalemci, E., 2002, Ph.D. Thesis, University of California, San Diego

[Kalemci et al. 2004] Kalemci, E., et al., 2004, ApJ, 603, 231

[Lin et al. 2000] Lin, D., Smith, I. A., Böttcher, M., \& Liang, E. P., 2000, ApJ, 531, 963

[Main et al. 1999] Main et al., 1999, ApJ, 525, 901

[Molnar \& Mauche 1986] Molnar, L. A., \& Mauche, C. W., 1986, ApJ, 310, 343

[Predehl \& Klose 1996] Predehl, P., \& Klose, S., 1996, A\&A, 306, 283

[Predehl \& Schmitt 1995] Predehl, P., \& Schmitt, J. H. M. M., 1995, A\&A, 293, 889

[Rodriguez et al. 2004] Rodriguez, J., et al., 2004, ApJ, 612, 1018.

[Tomsick \& Kaaret. 2004] Tomsick, J. A., Kaaret, P., 2001, ApJ, 548, 401 